\title{
BMJ Open Impact of cardiac rehabilitation on mortality and morbidity in diabetic versus non-diabetic patients: protocol for a systematic review and meta-analysis
}

\author{
Emad Hanna Dababneh (D) , ${ }^{1,2}$ Sumanta Saha (D) , ${ }^{3}$ Linda Östlundh (D) , ${ }^{4}$ \\ Rami H Al-Rifai (D) , ${ }^{1}$ Abderrahim Oulhaj (D) ${ }^{1}$
}

To cite: Dababneh EH, Saha S, Östlundh L, et al. Impact of cardiac rehabilitation on mortality and morbidity in diabetic versus nondiabetic patients: protocol for a systematic review and meta-analysis. BMJ Open 2021;11:e047134. doi:10.1136/ bmjopen-2020-047134

- Prepublication history and additional supplemental material for this paper are available online. To view these files, please visit the journal online (http://dx.doi.org/10.1136/ bmjopen-2020-047134).

Received 21 November 2020 Revised 03 March 2021 Accepted 26 March 2021

Check for updates

(C) Author(s) (or their employer(s)) 2021. Re-use permitted under CC BY-NC. No commercial re-use. See rights and permissions. Published by BMJ.

${ }^{1}$ Institute of Public Health, College of Medicine and Health Sciences, United Arab Emirates University, Al Ain, UAE

${ }^{2}$ Academic Affairs, Tawam Hospital, Al Ain, UAE

${ }^{3}$ R. G. Kar Medical College, Kolkata, West Bengal, India ${ }^{4}$ National Medical Library, United Arab Emirates University College of Medicine and Health Sciences, Al Ain, Abu Dhabi, UAE

Correspondence to Dr Abderrahim Oulhaj; aoulhaj@uaeu.ac.ae

\section{ABSTRACT}

Background Cardiac rehabilitation (CR) decreases the morbidity and mortality risk among patients with cardiac diseases; however, the impact of CR on patients with diabetes remains underexplored. This is a protocol for a systematic review and meta-analysis methodology to explore if the effect of CR on mortality and morbidity is the same in patients with type 2 diabetes compared with patients without diabetes. Methods and analysis Interventional and non-interventional studies comparing the effect of $\mathrm{CR}$, for at least 1 month, on all-cause mortality and cardiovascular outcomes including fatal and non-fatal myocardial infarction, revascularisation and rehospitalisation in adults with cardiac diseases will be deemed eligible for inclusion. Studies published between 1990 and 2020 will be searched in PubMed, Embase, Cochrane, CINAHL, Scopus and in registries for randomised controlled trials. Eligible studies will be selected using the Covidence software, and their salient details regarding the design, population, tested interventions and outcomes of interest will be gathered. The quality of studies to be deemed eligible and reviewed will be assessed using the Cochrane Collaboration and National Heart, Lung, and Blood Institute's tools. The appraisal process will be based on the study design (interventional and noninterventional). In the meta-analysis step, the pooled effect of CR on the outcomes will be estimated. All meta-analyses will be done using the random-effects model approach (inversevariance method). $P$ and $p$ value of $\chi^{2}$ statistics will guide the heterogeneity assessment. Subgroup analyses will also be performed. The small study effect will be investigated by generating the funnel plots. The symmetry of the latter will be tested by performing Egger's test.

Ethics and dissemination The systematic review will use data from published literature; hence, no ethical approval will be required. Findings of the systematic review and metaanalysis will be published in peer-reviewed international journals and will be disseminated in local and international scientific meetings.

PROSPERO registration number CRD42020148832.

\section{INTRODUCTION}

Cardiovascular diseases (CVDs) are the leading cause of mortality and disability across the globe in both adult men and women. Annually, worldwide, more people die from CVDs than any other cause. ${ }^{1}$ In 2016, almost 17.9 million
Strengths and limitations of this study

- The protocol follows the Preferred Reporting Items for Systematic Review and Meta-Analysis Protocols (PRISMA-P) and the systematic review and metaanalysis will follow the PRISMA 2009 guideline.

- The database search strategy is developed and tested by an expert librarian.

- Screening of citations to be retrieved, full-text screening, data abstraction, and risk of bias and quality assessment will be performed independently by at least two expert reviewers.

- Heterogeneity in effect estimates will be resolved through subgroup and meta-regression analyses; otherwise, the included studies will be reported narratively.

- Limitations may include lack of uniformity in the reported effect estimates of association in the literature.

people died from CVDs, comprising $31 \%$ of all global deaths. ${ }^{1}$ It is estimated that around 23.6 million people will die from CVDs by $2030 .^{2}$

Cardiac rehabilitation (CR) is a complex multidisciplinary intervention offered to patients suffering from cardiac diseases including myocardial infarction (MI), cardiac failure, surgery of cardiac valves, coronary artery bypass surgery and percutaneous coronary intervention. ${ }^{34}$ It is a well-recognised secondary prevention intervention aiming to decrease the risk of morbidity and mortality, and to enhance the quality of life in patients with a history of CVD. Several leading health organisations including the American Heart Association, The National Institute for Health and Care Excellence, and The British Association for Cardiovascular Prevention and Rehabilitation endorse CR for patients with CVD. ${ }^{5-12}$ CR comprises an exercise programme, smoking cessation intervention, behavioural and nutritional counselling, immunisation and interventions to treat blood pressure, lipid levels, obesity and diabetes mellitus. ${ }^{3}$ 
Typically, the CR programme starts during in-hospital admission and carries on after discharge from the hospital. ${ }^{3}$

Several studies and meta-analyses have shown the beneficial effect of CR in reducing cardiovascular morbidity and mortality and in improving patients' quality of life. ${ }^{4} 1314$ A very recent systematic review and metaanalysis in patients with acute coronary syndrome, coronary artery bypass grafting or mixed populations with coronary artery disease has shown a significant decrease in mortality among patients who were referred to CR (HR $0.37,95 \%$ CI 0.20 to 0.69$).{ }^{15}$

Despite the evidence that CR is an effective intervention to reduce morbidity and mortality in patients with history of cardiac disease, contemporary literature does not provide enough information on the beneficial effect of CR among patients with type 2 diabetes mellitus (T2DM). Many studies have shown an increased risk for recurrent CVD events in patients with T2DM compared with those without T2DM. ${ }^{1617}$ A very recent study have shown that following MI, adults with T2DM had a higher risk for recurrent CVD events compared with those with prior CVD but without T2DM. The current 2018 American Heart Association/American College of Cardiology guideline also considers patients with T2DM to be at very high risk for recurrent GVD events. ${ }^{18}$

Investigating the effect of $\mathrm{CR}$ in this subpopulation of patients is then of great interest. Considering the substantial global burden of T2DM and its complex association with recurrent cardiac diseases, it is crucial to understand how CR might help in controlling all-cause mortalities and cardiovascular disabilities (eg, fatal and non-fatal MI, revascularisation and rehospitalisation) in patients with T2DM. In this protocol, we propose to run a systematic review and meta-analysis to investigate the effectiveness of CR in decreasing the risk of mortality and morbidity among patients with T2DM. The protocol also presents a systematic methodology to explore if the effect of CR on mortality and morbidity is the same in patients with T2DM compared with patients without T2DM.

\section{METHODS}

Our protocol follows the Preferred Reporting Items for Systematic Review and Meta-Analysis Protocols (PRISMA-P) 2015 statement. ${ }^{19}$ The prospective review is registered with the PROSPERO (registration no. CRD42020148832). ${ }^{20} \mathrm{~A}$ request to update the information about starting date and search date inclusion in the PROSPERO registration form has been submitted. The start date for the review will be December 2020 and the estimated date for completion is set to December 2021.

\section{Eligibility criteria}

\section{Inclusion criteria}

We will include interventional and non-interventional studies reporting direct and/or indirect comparison of effect sizes in patients with diabetes versus patients without diabetes. All studies should have reported calculated or calculable estimates of the association between the measured exposure(s) and the outcomes(s). The systematic review will be carried out using the $\mathrm{PECO}(\mathrm{T})$ approach (participants, exposure, comparator, outcome(s) and type of study). The PECO(T) provides the framework from which studies are identified and selected for inclusion. ${ }^{21}$

- Population: 18 years or older patients with or without T2DM who had experienced at least one cardiac event.

- Exposure: CR regardless of the type, frequency, intensity, duration and the location where CR was provided (eg, at home or at a specialised healthcare facility).

- Comparators: No CR.

- Outcomes: All-cause mortality and/or CVD events including fatal and non-fatal MI, revascularisation and rehospitalisation.

- Type of study: Interventional and non-interventional studies.

\section{Exclusion criteria}

Studies with no comparison groups or case series studies will be excluded. Crossover studies will be also excluded if the outcome of interest is not reported before the crossover occurs. Also, letters, editorials and conference abstracts (without full publication) will be excluded. We will also exclude (1) studies among pregnant women with cardiac diseases, patients with a heart transplant or patients with implanted defibrillators/pacemakers, where the reported estimates are not separable from the other population groups; and (2) studies with no calculated or no calculable quantitative estimates on the association between the measured exposure(s) and the outcomes(s).

\section{Search strategy and study selection}

We will search for papers published between January 1990 and December 2020 in PubMed, Embase, Cochrane, CINAHL and Scopus, irrespective of the language of publication and geographical origin. A search combination of the fields: 'Title', 'Abstract' and MeSH/Thesaurus will be applied to ensure the best possible information retrieval. Additional searches will follow using clinical trial registry websites and the bibliography of articles included in the proposed review. The search strategy will include terms based on the population, interventions compared and outcomes. Pre-searches in PubMed to identify search terms and develop a search string were conducted in June-November 2020 and the full search, including all selected databases, will be completed in December 2020. The draft of the preliminary search strategy for PubMed is available as supplementary material (online supplemental file 1 ).

On uploading the retrieved citations to the Covidence software and removing duplicates, at least two reviewers will independently screen these citations against the above-stated eligibility criteria. Full text of the eligible and potentially eligible studies will be thoroughly screened and assessed for final inclusion. The review authors will resolve disagreements in the study selection process by consensus. In the event of many studies reporting findings on the same population, the one with more relevant information will be chosen. Cabell's Predatory Report will be informed to verify 
the scientific status of the open access papers selected for the review. ${ }^{22}$

\section{Data abstraction}

Data on study and participant characteristics, interventions compared and outcomes of interest will be gathered in a predefined Excel sheet by at least two reviewers independently. Disagreement during the process of data abstraction will be resolved by discourse. Details of studies to be collected will include publication details (year and journal), study design and duration, trial ID (if a clinical trial), country and setting where the study was performed, single or multicentred, ethical clearance, participant consent and funding information. Details regarding participants in each study will include sex and age, frequency of outcome data along with the reason of missingness (if any), diagnosis received, relevant baseline characteristics like HbAlc and fasting blood glucose for patients with diabetes, comorbidities and traditional risk factors. Regarding CR exposure, we will collect CR delivery-related details including its location, intensity/ frequency, duration, type, referrer, provider and financial support received (eg, medical insurance programme). For those who did not receive CR, details of any other intervention received will be gathered. For the outcome, depending on the data reported in the studies, we will collect the raw data, the estimated effects or both.

In studies reporting calculated crude and/or adjusted effect estimates of the respective outcomes of interest, these estimates will be extracted along with their corresponding 95\% CI limit. In studies with no calculated effect estimates, relevant data will be extracted to calculate the crude and/or adjusted estimates. A prioritisation scheme will be followed in extracting quantitative data. Within the same study, effect estimates stratified according to comorbidity will be prioritised, followed by age and duration of CR. Otherwise, the estimated overall effect will be used. One stratification level per included published research report will be considered to avoid double-counting. Stratified precalculated crude effect estimates (eg, stratified by age) will be extracted to produce one adjusted (eg, age-adjusted) effect estimate. In studies reporting adjusted effect estimates using multiple models, we will extract data from the model adjusted for the most number of confounders.

\section{Risk of bias assessment}

Using the Cochrane Collaboration tool, the risk of bias (RoB) of the randomised controlled trials will be evaluated for selection bias, performance bias, attrition bias, reporting bias and other biases. ${ }^{23}$ The selection bias will be assessed by evaluating the random sequence generation method used and the mechanism applied to conceal the sequence from the participants. The performance bias will be judged by assessing the mechanism and its adequateness concerning the blinding of study participants and personnel towards the intervention. For attrition bias, we will primarily evaluate the balance and reasons for missing data on the outcomes. Finally, reporting bias will be assessed by comparing the reported and unreported findings of each trial for any systematic differences.
Any additional bias not meeting any of the above types will be labelled as other biases. The risk of each of these biases will then be categorised as low, high or unclear.

Study Quality Assessment Tools by the 'National Heart, Lung, and Blood Institute' will be used to appraise the noninterventional studies, and the quality of each of these studies will be graded as low, high or unclear. ${ }^{24}$ Two reviewers will separately conduct the RoB assessment. Discrepancies will be resolved by discourse. If this discourse is not conducive, a third-party opinion will be sought.

\section{Evidence synthesis}

The pooled effect estimates of the respective outcomes will be estimated by meta-analysis using a random-effects model (Mantel-Haenszel method), as substantial clinical heterogeneity is anticipated in this kind of studies. This heterogeneity can be caused by the variability in participants' characteristics across studies (eg, diagnosis, the severity of cardiac diseases or T2DM, comorbidities, ethnicity, socioeconomic background and other risk factors), variability in interventions (eg, the period of time from cardiac event to CR initiation, the CR duration, frequency and intensity, the level of expertise among CR providers, and the place where CR therapy was given (eg, healthcare facility vs home-based), and variability in treatment modalities).

When raw dichotomous data are available for metaanalysis, the summary effect will be estimated in the risk ratio (RR). For meta-analysis with such studies, the interventionspecific frequency of the outcomes will be summed when it is available from multiple treatment arms. If an outcome event does not occur in one of the two treatment arms compared in a study, 0.5 will be added to each cell of the $2 \times 2$ table for meta-analysis (continuity correction). A study will be eliminated from the meta-analysis when this happens to both of its juxtaposed intervention arms. Such excluded studies will be included in the meta-analysis while estimating the pooled risk differences for sensitivity analysis.

Precalculated and calculated crude effect estimates will then be pooled using the random-effects meta-analysis model for calculating the crude summary effect. The meta-analysis will accompany a predictive interval estimation to gauge the intervention effects on the outcomes in future studies.

Due to the dichotomous nature of the outcomes, we anticipate a lack of uniformity in the reported measures of association in the literature. In some studies, the crude effect estimate might be quantified in the form of the OR, while in other studies, it might be quantified in the form of RR or HR. To obtain a unified and consistent crude summary effect estimate, OR will be converted into RR, as long as enough information on the baseline risk is provided in the primary study. Conversion of OR into an RR will follow a standard procedure using the following mathematical formula ${ }^{2526}$ :

$$
\mathrm{RR}=\mathrm{OR} /\left(1-\mathrm{p}_{0}+\left(\mathrm{p}_{0} \times \mathrm{OR}\right)\right)
$$

Where $\mathrm{p}_{0}$ is the baseline risk.

We are unaware of any mathematical equation that converts HR into RR, hence crude HRs will be pooled separately. As for the adjusted estimates of association, meta-analysis will be 
performed separately according to the type of the adjusted estimate. This means that the adjusted ORs, adjusted RRs and adjusted HRs will be pooled separately. To avoid bias and provide precise effect estimates, we will not combine crude with adjusted estimates. Furthermore, the meta-analysis will be performed separately for direct comparisons and indirect comparisons. More specifically, comparable effect estimates (eg, OR) obtained from direct studies will be pooled with those obtained from indirect studies according to diabetes status. The success of this plan will, however, be contingent on the quality and the type of effect estimates reported in the primary studies.

Forest plots will be used to depict the meta-analytic comparisons graphically. The statistical significance of meta-analysis findings will be determined at $\mathrm{p}<0.05 \%$ and $95 \%$ CI. Using the Grading of Recommendations Assessment, Development and Evaluation (GRADE) approach (by GRADE Working Group (2004)), ${ }^{27}$ the evidence quality of the outcomes varying statistically significantly between the juxtaposed interventions will be determined.

\section{Statistical heterogeneity and subgroup analysis}

Statistical heterogeneity across studies will be assessed using the $I^{2}$ statistics and the p value of $\chi^{2}$ statistics. The $I^{2}$ statistics of $25 \%, 50 \%$ and $75 \%$ will be categorised as low, moderate and high heterogeneity, respectively. ${ }^{28} \mathrm{~A} p$ value of $<0.1$ will be considered as a statistically significant heterogeneity. ${ }^{23} \mathrm{~A}$ subgroup analysis will follow if a meta-analysis of 10 or more studies depicts substantial statistical heterogeneity. ${ }^{23}$ The between-subgroup heterogeneity will be determined by a fixed-effects model meta-analysis.

We will subgroup by comorbidities, gender, age, CR characteristics and country income groups. A previously published meta-analysis showed that the referral to CR programmes was higher in men $(45 \%)$ compared with women $(38.5 \%)^{29}$; therefore, to understand how the effect estimates might vary between males and females, a gender-wise categorisation will be done. Research further suggests that poor CR programme participation can be influenced by rurality and poverty; therefore, using the World Bank income groups, we will subgroup the studies as low-income, lower-middle-income, uppermiddle-income and high-income nations. ${ }^{30}$ Next, a homebased CR programme is expected to improve participation and outcome, especially in patients who live distant from the specialised CR services. ${ }^{3}$ A Cochrane review found that homebased CR services might help to ensure better adherence, participation and outcomes than CR provided in specialised centres. ${ }^{31}$ Therefore, we will dichotomise studies according to whether CR was home-based or was provided in specialised centres, and see how the effect size varies accordingly. Also, we will investigate the effect of CR based on the source of information, whether the source was based on information received through over-phone consultations or from social media channels. These services are new areas of interest and telephone-based counselling has been suggested to be beneficial in reducing hospitalisation, improvement in smoking cessation rates, blood pressure control, and depression and anxiety scores. ${ }^{332}$

\section{Publication bias and sensitivity analysis}

We will assess for small study effect visually using funnel plots and contour enhanced funnel plots, and statistically by Egger's test when at least 10 studies will be available for meta-analysis. ${ }^{23}$

We will proceed with the following types of sensitivity analyses to examine how the effect estimates differ in various hypothetical situations. First, we will repeat our meta-analysis using a fixed-effects model approach. Then, we will determine the difference in OR and RR estimates. Additionally, for the statistically significant risk differences, we will calculate the number needed to treat. Moreover, when our preliminary analysis incorporates adjusted estimates in the meta-analysis, whenever available, the sensitivity analysis will repeat the analysis using the unadjusted estimates. Additionally, stratum-wise meta-analysis will be done based on the following CR service-related features-duration, location (eg, home vs institution), financial support (eg, if CR service covered by insurance or not) and type of the cardiac event for which CR was prescribed.

\section{Handling missing data}

For statistically significant meta-analytic estimates that are based on raw data from at least 10 studies, the imputation case analysis will ensue to determine the impact of missing data. We will use complete case analysis as our reference and compare its results with that of the subsequent analyses worst-case scenario, best-case scenario, and Gamble and Hollis analysis. ${ }^{33}$

All analyses will be done using $\mathrm{R}$ and Stata statistical software V.16 (StataCorp, College Station, Texas, USA). When the quantitative analysis is not possible, findings will be reported narratively.

\section{Patient and public involvement}

No patient involved.

\section{Ethics and dissemination}

This is a protocol for a systematic review and metaanalysis; hence, ethical approval is not needed. Findings of the systematic review and meta-analysis to be carried out following this protocol will be published in peerreviewed international journals and will be disseminated in local and international scientific meetings.

Contributors EHD and AO conceptualised the research question. EHD, LÖ, SS, RHA and $\mathrm{AO}$ contributed equally to protocol drafting and writing. RHA and $A O$ designed the analysis plan, critically revised the protocol and approved the final version. All authors read and approved the final manuscript.

Funding The authors have not declared a specific grant for this research from any funding agency in the public, commercial or not-for-profit sectors.

Competing interests None declared.

Patient consent for publication Not required.

Provenance and peer review Not commissioned; externally peer reviewed.

Data availability statement This is a protocol for a systematic review and metaanalysis. No data is available to be shared at this stage.

Supplemental material This content has been supplied by the author(s). It has not been vetted by BMJ Publishing Group Limited (BMJ) and may not have been peer-reviewed. Any opinions or recommendations discussed are solely those 
of the author(s) and are not endorsed by BMJ. BMJ disclaims all liability and responsibility arising from any reliance placed on the content. Where the content includes any translated material, BMJ does not warrant the accuracy and reliability of the translations (including but not limited to local regulations, clinical guidelines, terminology, drug names and drug dosages), and is not responsible for any error and/or omissions arising from translation and adaptation or otherwise.

Open access This is an open access article distributed in accordance with the Creative Commons Attribution Non Commercial (CC BY-NC 4.0) license, which permits others to distribute, remix, adapt, build upon this work non-commercially, and license their derivative works on different terms, provided the original work is properly cited, appropriate credit is given, any changes made indicated, and the use is non-commercial. See: http://creativecommons.org/licenses/by-nc/4.0/.

\section{ORCID iDs}

Emad Hanna Dababneh http://orcid.org/0000-0002-9895-5379

Sumanta Saha http://orcid.org/0000-0003-0996-8846

Linda Östlundh http://orcid.org/0000-0001-5091-604X

Rami H Al-Rifai http://orcid.org/0000-0001-6102-0353

Abderrahim Oulhaj http://orcid.org/0000-0002-5330-904X

\section{REFERENCES}

1 World Health Organization. Cardiovascular diseases (CVDs), 2017. Available: https://www.who.int/en/news-room/fact-sheets/detail/ cardiovascular-diseases-(cvds) [Accessed 16 June 2020].

2 World Health Organization (no date) WHO. About cardiovascular diseases. Available: https://www.who.int/cardiovascular_diseases/ about_cvd/en/ [Accessed 16 June 2020].

3 Servey JT, Stephens M. Cardiac rehabilitation: improving function and reducing risk. Am Fam Physician 2016;94:37-43.

4 Dalal HM, Doherty P, Taylor RS. Cardiac rehabilitation. BMJ 2015;351:h5000.

5 Anderson L, Thompson DR, Oldridge N. Exercise-based cardiac rehabilitation for coronary heart disease. Cochrane Database Syst Rev 2016;1:CD001800.

6 Goel K, Lennon RJ, Tilbury RT, et al. Impact of cardiac rehabilitation on mortality and cardiovascular events after percutaneous coronary intervention in the community. Circulation 2011;123:2344-52.

7 Goel K, Pack QR, Lahr B, et al. Cardiac rehabilitation is associated with reduced long-term mortality in patients undergoing combined heart valve and CABG surgery. Eur J Prev Cardiol 2015;22:159-68.

8 Suaya JA, Stason WB, Ades PA, et al. Cardiac rehabilitation and survival in older coronary patients. J Am Coll Cardiol 2009;54:25-33.

9 Norris CM, Jensen LA, Galbraith PD, et al. Referral rate and outcomes of cardiac rehabilitation after cardiac catheterization in a large Canadian city. J Cardiopulm Rehabil 2004;24:392-400.

10 Alter DA, Oh PI, Chong A. Relationship between cardiac rehabilitation and survival after acute cardiac hospitalization within a universal health care system. Eur J Cardiovasc Prev Rehabil 2009;16:102-13.

11 Martin B-J, Hauer T, Arena R, et al. Cardiac rehabilitation attendance and outcomes in coronary artery disease patients. Circulation 2012;126:677-87.

12 Giannuzzi P, Temporelli PL, Marchioli R, et al. Global secondary prevention strategies to limit event recurrence after myocardial infarction: results of the gospel study, a multicenter, randomized controlled trial from the Italian cardiac rehabilitation network. Arch Intern Med 2008;168:2194-204.
13 Anderson L, Taylor RS. Cardiac rehabilitation for people with heart disease: an overview of Cochrane systematic reviews. Cochrane Database Syst Rev 2014;12:CD011273.

14 Shepherd CW, While AE. Cardiac rehabilitation and quality of life: a systematic review. Int J Nurs Stud 2012;49:755-71.

15 Salzwedel A, Jensen K, Rauch B, et al. Effectiveness of comprehensive cardiac rehabilitation in coronary artery disease patients treated according to contemporary evidence based medicine: update of the cardiac rehabilitation outcome study (CROSII). Eur J Prev Cardiol 2020;27:1756-74.

16 D'Agostino RB, Russell MW, Huse DM, et al. Primary and subsequent coronary risk appraisal: new results from the Framingham study. Am Heart J 2000;139:272-81.

17 Heinl RE, Dhindsa DS, Mahlof EN, et al. Comprehensive cardiovascular risk reduction and cardiac rehabilitation in diabetes and the metabolic syndrome. Can J Cardiol 2016;32:S349-57.

18 Grundy SM, Stone NJ, Bailey AL, et al. 2018 AHA/ACC/AACVPR/ AAPA/ABC/ACPM/ADA/AGS/APhA/ASPC/NLA/PCNA Guideline on the Management of Blood Cholesterol: Executive Summary: A Report of the American College of Cardiology/American Heart Association Task Force on Clinical Practice Guidelines. J Am Coll Cardiol 2019;73:3168-209.

19 Moher D, Shamseer L, Clarke M, et al. Preferred reporting items for systematic review and meta-analysis protocols (PRISMA-P) 2015 statement. Syst Rev 2015;4:1.

20 Dababneh E, Oulhaj A, AL-Rifai RH. Impact of cardiac rehabilitation on mortality and morbidity in diabetic versus non-diabetic patients: a systematic review and meta-analysis. Available: https://www.crd. york.ac.uk/PROSPERO/display_record.php?RecordID=148832

21 Woodruff TJ, Sutton P. The navigation guide systematic review methodology: a rigorous and transparent method for translating environmental health science into better health outcomes. Environ Health Perspect 2014;122:1007-14.

22 Cabell's Scholarly Analytics. Cabell's Predatory Report. Cabell's Scholarly Analytics, 2020. Available: https://www2.cabells.com/ about-predatory

23 Higgins J, Thomas J. Cochrane Handbook for systematic reviews of interventions. 6ed. The Cochrane Collaboration, 2019.

24 Study Quality Assessment Tools. National heart, lung, and blood Institute. Available: https://www.nhlbi.nih.gov/health-topics/studyquality-assessment-tools

25 Grant RL. Converting an odds ratio to a range of plausible relative risks for better communication of research findings. BMJ 2014;348:f7450.

26 Shrier I, Steele R. Understanding the relationship between risks and odds ratios. Clin J Sport Med 2006;16:107-10.

27 Atkins D, Best D, Briss PA, et al. Grading quality of evidence and strength of recommendations. BMJ 2004;328:1490.

28 Higgins JPT, Thompson SG, Deeks JJ, et al. Measuring inconsistency in meta-analyses. BMJ 2003;327:557-60.

29 Samayoa L, Grace SL, Gravely S, et al. Sex differences in cardiac rehabilitation enrollment: a meta-analysis. Can J Cardiol 2014;30:793-800.

30 The World Bank Group. WDI - Classifying countries by income, 2019 Available: https://datatopics.worldbank.org/world-developmentindicators/stories/the-classification-of-countries-by-income.html [Accessed 17 June 2020].

31 Anderson L, Sharp GA, Norton RJ, et al. Home-Based versus centre-based cardiac rehabilitation. Cochrane Database Syst Rev 2017;6:CD007130.

32 Kotb A, Hsieh S, Wells GA. The effect of telephone support interventions on coronary artery disease (CAD) patient outcomes during cardiac rehabilitation: a systematic review and meta-analysis. PLoS One 2014;9:e96581.

33 Gamble C, Hollis S. Uncertainty method improved on bestworst case analysis in a binary meta-analysis. $J$ Clin Epidemiol 2005;58:579-88. 